\title{
Design of advanced smart ultraluminescent multifunctional nanoplatforms for biophotonics and nanomedicine applications
}

\author{
Carina I Salinas ${ }^{1}$ and A Guillermo Bracamonte ${ }^{1,2 *}$ \\ ${ }^{1}$ Instituto de Investigaciones en Físico Química de Córdoba (INFIQC), Departamento de Química Orgánica, Facultad de Ciencias Químicas, Universidad Nacional \\ de Córdoba. Ciudad Universitaria, 5000 Córdoba, Argentina \\ ${ }^{2}$ Departement de chimie and Centre d'optique, photonique et laser (COPL), Université Laval, Québec (QC), G1V 0A6, Canada
}

\begin{abstract}
The design and synthesis of new Nanomaterials applied for Bio-applications as Nanosensors of single molecule detection, Biosensors of biological events and controlled drug delivery nanoparticles are of high interest and impact in bioanalytical methodologies, clinical research and as well as from new areas of research as Nanophotonics, Biophotonics, and Nanomedicine. First, one of the higher challenges within all these research fields is how to transduce an analytical signal and transduce it from individual biological events or biomolecules. And at the same time secondly, it should be tracked biological event during the time by a selective response followed by a given therapy action depending of needs. In order to afford these challenges the control at the nanoscale incorporating different types of materials from hybrids Nanomaterials to Metamaterials for variable and biocompatible properties for targeted applications. Due to the high impact, if it is tuned these properties based on specific requirements new therapies within Precision medicine are open. For these reasons, in this short Review was exposed different strategies for Smart tunable Nanoarchitectures as Nanoplatforms for Bioconjugation to design Lab. On-particle and Drug delivery Nano-systems accompanied with Luminescent and Ultraluminescent properties based on Fluorescence Resonance Energy Transfer (FRET), Metal Enhanced Fluorescence (MEF), Enhanced Plasmonics (EP) coupled to MEF as well as FRET coupled to MEF applied for early diagnosis, biomolecular and DNA detection, Genomics, Gene therapies and Drug Delivery Systems by the application of synthetic and Bio-inspired Nanoarchitectures based on Hybrids Nanomaterials. By this manner advanced treatments in-situ or in vivo after stimulation by a smart bio-response were discussed.
\end{abstract}

\section{Introduction}

Design and controlled synthesis at the nanoscale of different types of nanoparticles composed as well by a large number of materials opened their application within different areas of Life Sciences [1] All these nanomaterials are from inorganic materials as metallic, semiconductors, quantum particles, and conductive; magnetic as well as organic materials from small molecules to polymeric nanomaterials with variable properties depending of their applications [2].

Within Life Sciences exist a huge number of challenges and needs; and for these reasons new areas of Research related with Optical Nanomaterials joined to Optics produced in the last years an exponential number of advanced Research publications accompanied with applications by the introductions of available products for Life Sciences studies, early diagnosis based on Imaging; and new treatments that it could contemplate Genomics for Precision Medicine. For all these applications the control of the classical light and non-classical light from new nanoarchitectures of Nano-emitters is of high impact and at the same time it is a Research Field as well.

Metal Enhanced Fluorescence (MEF) is a phenomena related with an increased emission of fluorescence emission produced by an enhanced absorption of the fluorophores in the near field of metallic surfaces where it is generated stronger electromagnetic fields that increase the higher electronic excited state [3-5]. This phenomenon could be collected from metallic surfaces as well as from nanoparticles.
Moreover in presence of multiple numbers of individual nanoparticles that interact producing enhanced electromagnetic fields between nanoparticles [6], known as Enhanced Plasmonics [7] (EP) can be coupled to MEF in order to collect increased molecular detection within the near field [8] Core-shell nanoparticles formed by metallic cores covered with variable silica shells for fluorescent modification showed enhanced properties related with the MEF phenomena accompanied with diminished photobleaching properties with high potential impact on Life Sciences. In addition supramolecular modifications showed enhanced detection based on non-covalent host-guest interactions coupled to a switched on-off MEF by a simple chemical reaction [9] with potential applications for Single Molecule Detection (SMD) [10] This proof of concept developed by us it could be extended to molecular tracking and host-guest complexes delivery in biological media by applications of antibodies recognition [11] as well. For these reasons the design and synthesis of different Nanoarchitectures based on Supramolecular chemistry [12,13] Organic chemistry joined to inorganic chemistry as well have potentials application within

${ }^{\star}$ Correspondence to: A Guillermo Bracamonte, $\mathrm{PhD}$, INFIQC, Institute of Physicochemical Research of Cordoba, Department of Organic Chemistry, National University of Cordoba (UNC), Argentine, E-mail: gbracamonte@fcq.unc.edu.ar; guillermobrac@yahoo.ca

Key words: hybrids nanomaterials, nanophotonics, biophotonics, nanomedicine

Received: June 01, 2018; Accepted: June 14, 2018; Published: June 16, 2018 
Biomaterials sciences for targeted applications due to their versatilities and tunable properties [2] as it was demonstrated by a controlled switched on-off variation of molecular fluorescence [14] that produced variable resolution with potential application within NanoImaging and Bioimaging. However, the challenge of the fluorescence control from molecular to Nanoscale levels still existing needs to be improved and enhanced the light generation.

Moreover, the importance of light collection as fluorescent emission it was showed from individual nucleic acids tracking by DNA fluorescent labelling by microfluidics coupled to Laser Fluorescence Microscopy Imaging analysis [15], as well as by single DNA strands detection with the application of fluorescent polymers [16] and intercalant agents [17].

All these mentioned Research fields can be applied for challenges related with Advanced issues within Genomics [18] and Precision Medicine [19,20], as for example for genotyping and targeted treatments, with potential application in epigenetics for non-canonical 3D DNA structures [21] as well. In addition to develop controlled sizes of particles at the nanoscale accompanied with smart responses it should be tuned each part of the Nanoarchitecture.

In this short Review was discussed the application of different Nanomaterials to develop hybrids Nanoparticles for Advanced Smart Ultraluminescent Multifunctional Nanoplatforms with applications within Biophotonics and Nanomedicine.

\section{Smart hybrids nanocomposites}

Different Nanoarchitectures by varying Nanomaterial composition could be obtained in order to be applied as Nanoplatforms of chemical functionalization for targeted applications. Moreover, these nanoparticles accompanied with variable composition and surface functionalization could act as responsive single dots that collect, transduce, enhance signals for tracking applications and produce specific actions depending of stimulations given by the environment. For the design of these Smart Nanomaterials should be combined different material properties coupling variable functions from each component added to finally obtain Meta-materials [22] and hybrids Nanoarchitectures [2]. In addition to all these requirements another challenge should be added within Life Sciences related with the Biocompatibility and toxicological issues [23] as well.

For Nanoplatforms design in colloidal dispersion it should be began from a template at the Nanoscale in order to add variable composition on it. By this manner variable templates could be applied going from inorganics Nanomaterials, as Aluminium [24], Indium [25], Cooper [26], Gold, Silver [27,28] with variable sizes and shapes; Organic materials, as Molecular spacers [29], lineal and branched Polymers [30] and Supramolecular systems [31,32] and Biological materials as antibodies [29], biomolecules [29], etc.. The deposition and chemical modification of the Nano-template will permit tuneable properties of the nanoparticle. For example bi-metallic Core-shell nanoparticles [33], metallic Core-shell polymeric silica nanoparticles [34], metallic Core-shell supramolecular nanoparticles [9], Polymeric Core-shell nanoparticles [35], etc.

These nanoparticles as Nanoplatforms accompanied with the right chemical modification showed to be Smart Nanosystems that produced variable responses depending of the Nanomaterial compositions as for example; fluorescence emission enhancement based on MEF [36], $\mathrm{pH}$ sensing based on the modification of the MEF signalling [37], fluorescent complex delivery tracked by enhanced fluorescence [9] Bioimaging with potential applications in Biodetection [38], drug delivery coupled to Imaging [39]; opening by this manner diverse applications as well as potential developments in many fields (Figure 1).

\section{Nano-biomaterials}

Nanotechnological developments were produced in the last years at different levels accompanied with many discussions related with environmental implications, health risks, toxicology and ethical issues from the manipulation to the applications in real samples [40]. But for the real application of Nanosensors, Biosensors and Nano-cargo for drug delivery in vivo, the first point to be taken into account is the Biocompatibility at first contact as well as at long term the bioaccumulation.

By this manner gold nanoparticles showed biocompatibility from small animals to non-human primates [41] and bio-applications [42], silver nanoparticles anti-microorganisms activity [43], biodegradable nanoparticles formed by variable synthetic and natural co-polymers based on hydrolysable organic functional groups [44].

And at this level the design by Bio-inspiration it should be applied and assayed in order to apply as for example lineal polymers of Polyethylene glycols (PEG) molecules of variable lengths to avoid Nanoaggregation with Bio-microstructures as cells and applied for nanoparticles synthesis [45]. And then, these nanomaterials were evaluated as for example based on the analysis of their hydrophilicity and hydrophobic domains evaluating the Mitigation of Inflammatory Immune Responses [46]. By this manner, it could be highlighted the Cyclodextrins supramolecular structures formed by glucose (cyclic oligosaccharides), that they were applied for conducting the first inhuman phase I clinical trial involving the systemic administration of siRNA to patients with solid cancers using a targeted, nanoparticle delivery system [47].

So, for Lab-on particles developments, Nano-cargo for drug delivery, Ultraluminescent Nanoplatforms for Bioimaging; and surface Nanoparticle Bioconjugations; it should be contemplated many Biocompatibilities issues for in vivo applications that add an additional challenge and Research work.

\section{Biocompatible and Ultraluminescent gold Core-shell Nanoparticles}

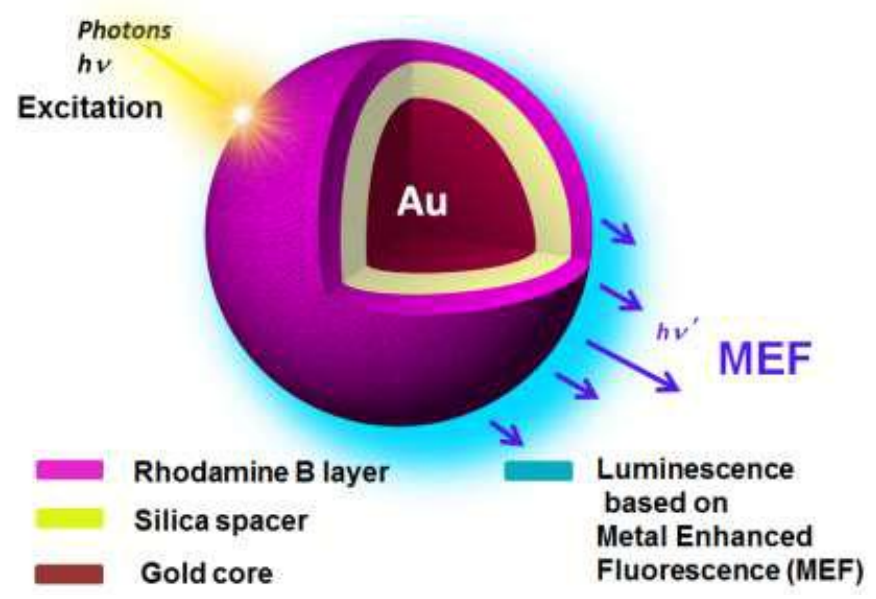

Figure 1. Scheme of Biocompatible and Ultraluminescent gold Core-shell nanoparticles (Au@SiO2-RhB) based on Metal Enhanced Fluorescence (MEF). Reprinted with permission from Ref 38 (Guillermo Bracamonte et al.). Copyright 2017 RSC Adv 


\section{Precision medicine}

In the last years the most important discussion about Medicine came from Cellular Biology, Biomolecular studies and Genomics Research [48] from where it was generated many concepts and new ideas towards targeted and specific treatments [49]. And within this field the higher impact came from human genome studies [50]; phenotypes and Epigenetics [51], due to that all the modifications produced different biomolecular and structural variations accompanied with variable biological compositions and functionalities as well. In this way, it should be highlighted based on the genomic knowledge a gene therapy by the embryonic cell modifications with CRISP (clustered regularly interspaced short palindromic repeats) [52]. This technique was based on enzymatic scissors that permitted to modify the genetic code by the correction of $\beta$-thalassemia mutant related with hemoglobin production disorders [53]. Moreover, it should be highlighted as well the importance of the recently application of gold nanoparticles as Nanoplatforms and Nano-carriers for targeted Delivery of CRISPRCas9 Therapeutics [54].

By this way, many other examples could be cited; but it is mentioned, due their importance, the gene related with the intolerance of the lactose [55]. This gene was produced by random mutations occurred in regions upstream of the LCT gene depending of variable phenotypes patterns depending of regions of all around the world. By this manner, an extra challenge could be added for faster analysis and early diagnosis based on new and faster Bioimaging assays [56].

\section{Biological signalling tracking by enhanced fluorescence methodologies}

From Physics, Optics, Nanophotonics and Biodetection Research studies were afforded many Research challenges of Biology as internal molecular cells signals as light emissions that opened new Research fields as Biophotonics.

Fluorescence Resonance Energy Transfer [57] (FRET) have already developed and studied for molecular sensing and applied for Biophotonics [58] applied to biological signal tracking. As it is known FRET by individual molecules interactions showed a short length effect related with energy transfer. In addition, the overlapping of donoracceptor spectroscopical characteristics was required, as well as the quantum yields of both emitters to low signals recording. In order to overcome these low signalling it should be highlighted the FRET coupled to enhanced fluorescence techniques as Metal Enhanced Fluorescence (MEF) by Multi-layered silver Core-shell nanoparticles [59] with the incorporation of a fluorescent positively charged Polymer and silica shell; as well as between inter-nanoparticle dimmers linked by complementary DNA strands [60] developed by Boudreau and Lakowicz et al. respectively; where the short distance at the nanoscale should be accurately controlled [61]. However, from Nanophotonics new studies based on confined molecules showed as well as long distances energy transfer effect [62] between electromagnetic couplings of them with potential Bio-applications.

All these studies demonstrated the versatility and application of these phenomena between individual molecules positioned at the right distance to transduce fluorescence energy signals. For these reasons FRET was applied by different techniques, as for example microscopy imaging of live cells for protein localizations [63], by Cytometry it was even studied molecular interaction within cells [64] and by Fluorescence Lifetime Imaging Microscopy (FLIM) [65] it was arrived to studies of protein-protein interactions. At the same time, in order to understand limit of detections, the importance of statistics and data collection related with non-radiative pathways via FRET from individual molecules are very important too [66]. By this manner there are still existing challenges and needs to be afforded due to the low concentrations present in nature for in vivo applications for Single Molecule Detection (SMD) [67] techniques. As for example, it could be mentioned from Bacterial interne molecular signalling [68] and special requirements and challenges for sensing in vivo and real samples with Nanosensors [69].

Moreover, it should be mentioned the high impact based on advances of new Photonic Nano-materials based on biomolecules as DNA and RNA joined to FRET phenomena [70] with potential real applications within genomics [71]. So, depending of the research directions of the design of new Nanomaterials will be as well the impact on the availability of new tools for Nanomedicine and Research [72].

\section{Metal enhanced fluorescence (MEF), FRET and coupled methodologies}

The Ultra luminescent properties are based on a plasmonic effect named Metal Enhanced Fluorescence (MEF). Metal enhanced fluorescence (MEF) is a plasmonic effect that enhances the emission fluorescence of a substrate placed at a given distance from a metallic surface [73]. The MEF effect depends of the distance of the fluorophore from the metallic surface due to the electromagnetic field intensity decays exponentially $\left(1 / \mathrm{r}^{3}\right)$ affecting by this manner drastically the fluorophore excitation [74]. For this reason in order to evaluate this parameter there are many studies developed using polymeric spacers as silica. In these Nanoarchitectures, the fluorophore is covalent bonded and the concentration can be controlled for maximal enhancements. These studies are in progress over surfaces [75] and colloidal dispersions [76], depending of the Nanoarchitecture design and applications. The main parameters to be controlled for optimal enhancements are the following: a) plasmonic complementarity of the Nanoparticle with the fluorophore, b) the distance and position of the dipolar momentum of the fluorophore from the metallic surface, c) the concentration of the fluorophore and d) the Nano aggregation state. Each of these parameters should be studied for optimal properties depending of the targeted property and application.

About Medical Imaging based on fluorescent techniques can be mentioned fluorescence molecular tomography, where based on this technique many developments were done in order to generate images in vivo using specific fluorophores [77] In addition from the viewpoint of NanoImaging with potential application, there are more developments done for cancer detection [78], cell targeting [79], and membrane characterization [80]; where the resolution it is an important factor to control and tune depending of the nanoscale of application that can be from a few molecules aggregates [81] to membranes and higher structures [82]. For this reason the relevance of the control of the nanostructure size and luminescent properties it is an important factor in order to design multifunctional nanoparticles applied to diagnostics and Nanomedicine based on Nano Imaging in vivo, where it is essential a) targeting, b) detection, c) tracking, c) stability of the signal, d) and activation of additional switchable function depending of the environment. So, as it was discussed the classical, non-classical and bio-light or luminescence generated and transduced was highly dependent of inter-emitter distances as well. For all these reasons there are large number different Research fields focussed on Optics and resolution imaging, Biostructures detection and characterisation, and accurate synthesis at the Nano-scale of variable material compositions. 


\section{Nanophotonics for biophotonics and early diagnosis}

Nanophotonics Research based on Nanomaterial in colloidal dispersion as well as deposed over modified surfaces, focussed on Bioapplications are in current development affording different challenges of high impact that contemplated from small Bio-molecules tracking, DNA, Cells, Bacteria and Biostructures detections for early diagnosis. In addition the detection should be accompanied with advanced and personalized treatments with tracking at long terms for optimal results. By this manner these fields are on the frontiers of chemical, drugs and personalized Nanomedicine.

From these fields not only it will generated detection and tracking of Biological events; but as well it will be contemplated a total control of all the environment of study in order to regulate the normal functionalization or even improve it for specific objectives of higher level that will produce, activate and modify the original bio-functions.

In this way, based on the Nanoscale and MEF control many publications were reported recently contemplating variable Nanotechnological applications [83] by the design and synthesis of Ultraluminescent Nanoplatforms of Core-shell nanoparticles for small biomolecules detection [84], Multi layered silver Core-shell nanoparticles for enhanced detection of DNA [85], that generated impact on new Research fields as for example Metal-Enhanced Fluorescence in Plasmonic Waveguides by Boudreau et al. [86], and smart responsive low volume conical 96 well plates for enhanced Fluorescence applications, accompanied with MEF enhancements of 100 , with Patent pending, based on surface modifications by Geddes et al. [87] (Figure 2).

In addition, the detection of different types of bacteria is highly required in Bioanalytical Chemistry, Clinical Chemistry, and environmental chemistry due to their high impact in different fields as Biodetection for different reasons. By this manner it was applied Ultraluminescent and biocompatible gold Core-shell nanoparticles previously developed by Bracamonte et al., based on MEF [38], for individual Escherichia Coli bacteria labelling and detection by Laser Fluorescence Microscopy [88] (Figure 3).

Moreover, as it was previously discussed within Biophotonics is highly desired individual biomolecules detection for early diagnosis. In this way, it should be mentioned Single Molecule Detection (SMD) Nanoplatforms developed by Bracamonte et al. [89] based on MEF
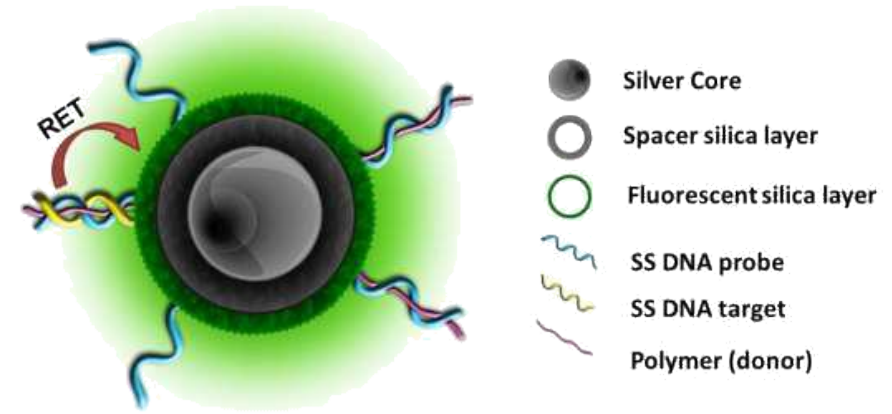

Figure 2. Fluorescent multilayer core-shell NPs architecture for DNA detection in three steps: 1) target-ready NPs are prepared by complexing ssDNA probe-grafted NPs with a fluorescent polymer transducer; 2) hybridization of target DNA with ssDNA probes activates the polymer transducer as energy donor toward dye-doped silica shell and excitation at $410 \mathrm{~nm}$ generates fluorescence emission by acceptor molecules at $550 \mathrm{~nm}$. Reprinted with permission from Ref 85 (Denis Boudreau et al.). Copyright 2014 Hindawi. Advances in Chemistry

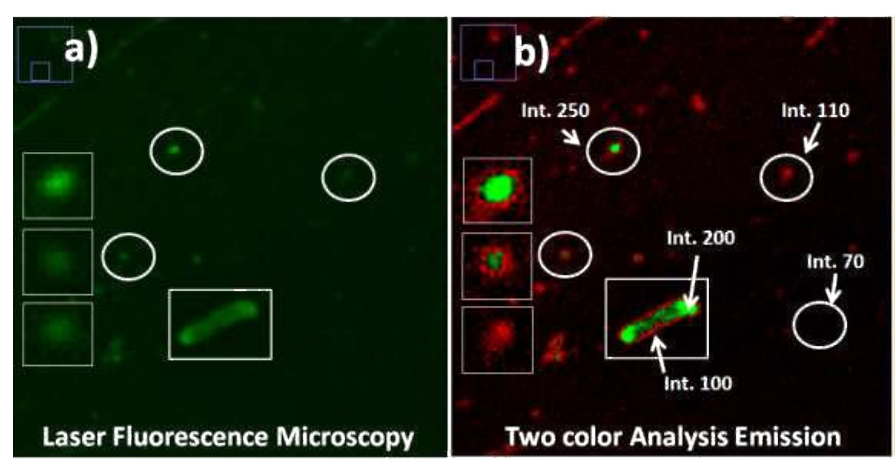

Figure 3. Biodetection of individual Escherichia coli bacterium: a) Fluorescence microscopy of individual Escherichia coli bacteria labelled with luminescent gold-core shell nanoparticles (image edited with green colour LUT). b) Image edited by two-colour analysis with red-green LUT. The insets are amplification of luminescent Core-shell Nanoparticles indicated with white circles (Intensities $\times 10^{2}$ ). Reprinted with permission from Ref 88 (Guillermo Bracamonte et al.). Copyright 2018 J. of Nanophotonics

(Figure 4) and Enhanced Plasmonics coupled to MEF in progress as well. Further Research work it should be done in order to improve and apply these types of Nanoarchitectures Bioconjugated with antibodies for In Flow methodologies coupled to different Optical techniques; and in-vivo biomolecular tracking by Laser Fluorescence Microscopy as well.

\section{Nanomedicine based of bioimaging, genomics and drug delivery systems}

New treatments related with different health problems as well as improvements in the functionalities of many metabolic pathways by a targeted Nano-cargo drug delivery systems that produce a response just only against media modifications is and it will be a challenge to overcome. In addition early diagnosis of special needs depending of individual constitutions open the Genomics knowledge that it is a high impact field from all the constitutive matter of humans is generated; and after the decoding of the whole genome its looks like it should be applied. However, still existing needs and challenges in many other fields from where it will be taken knowledge to be part of early diagnosis and specific treatment required.

As example it could be described, a label free DNA Biosensing based on a multilayered fluorescent nanocomposite and a cationic polymer that interact specifically with the DNA strands showing an enhanced quantum yield in presence of the double strands developed by Boudreau et al.. This activated energy of the fluorescent polymer after the complementary DNA interaction was coupled to MEF by a silver Core-shell nanoparticle as energy acceptor that produced bright and strong spot within in flow methodology coupled to Laser Fluorescence detection [90]. Then, this proof of concept, was applied to the Direct molecular detection of SRY gene from unamplified genomic DNA by metal-enhanced fluorescence and FRET [91] (Figure 5) and validated in real samples of blood [92] as well. This example was applied to the gen SRY but it could be developed to another gens depending of needs.

In addition, from Metabolomics based on the gene expression as well it is required the detection of different biomolecules as proteins. Within this field it could be mentioned the development done by Koenig et al. for peptides and proteins detection based on a Luminescent vesicle formed by fluorescent energy acceptors incorporated near protein recogniser sites given by supramolecular systems [93] as well. Then, this proof of concept was applied to thrombin [94] detection in blood real samples. 
Moreover, as it was mentioned previously to the Biocompatibility of the Nanomaterials it should be tuned specific properties for tracking applications. For example Biocompatible Ultra luminescent gold Coreshell Nanoplatforms for multifunctional applications developed by Bracamonte et al. [38] and applied to Ultraluminescent Escherichia Coli Biolabelling [88]; it could be mentioned.Then, they were tuned at different sizes and by this manner variable Nano-resolution were obtained; that produced detail of bacteria structures as well. From these controlled luminescence at the nanoscale potential applications it could be developed for non-canonical forms of DNA imaging and by this manner new approaches of Nanoplasmonics materials for Biosensing and Enhanced Light-Matter Interactions [95].

After the early diagnosis, where the time and costs are fundamental in order to expand their application it should be applied targeted treatments; and there it should be mentioned all the targeted Nanoarchitectures cargo for drug delivery applications. To this functionality it should be added extra functionalities and by this manner design and synthesis of Multifunctional Nanocomposites.

Cargo delivery Nano-systems of drugs, biomolecules, DNA and RNA, enzymes for controlled dosification depending of need and specific treatments should be developed in the next years. Enzymatic protection by Nano-carriers as for example CRISPR, controlled delivery of SiRNA, antibiotic administrations, and enhanced luminescent tracking of Cargo Nanoparticles and controlled drug delivery accompanied by Imaging methodologies developments, and joined to Optical activation as well. It could be mentioned as for example, from recently published articles, a smart responsive insulin administration against glucose [96], self-assembled gold nanoclusters for Bright Fluorescence Imaging and Enhanced Drug Delivery combining plasmonic and polymeric properties, only modifying the $\mathrm{pH}$ of the medium [97] Cargo Nanoparticles tracking [98], and quantifying drug uptake in skin by fluorescence [99]. And just to finish it should be highlighted the impact of Optical developments in these Research fields on the previous issues discussed, as for example Fluorescence Molecular Tomography (FMT) [100], as well as with non-invasive signalling tracking for Neurophotonics, Epigenetics and Bioimaging [101] (Figure 6).

a)

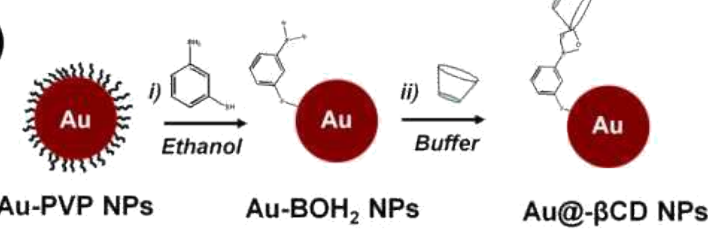

b)

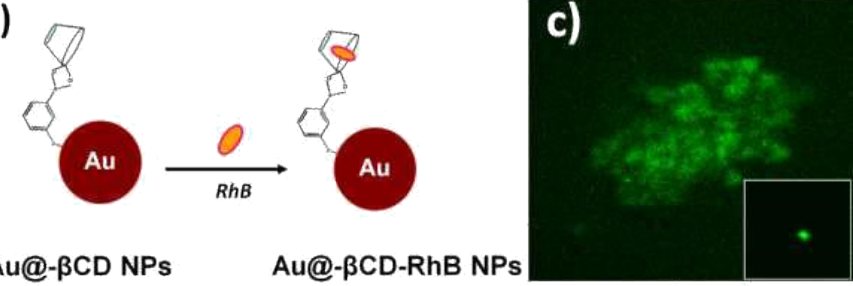

Figure 4. Single Molecule Detection (SMD) Nanoplatform: a) Schematic representation of Gold nanoparticles grafted with $\beta$ CD. b) Molecular recognition event based on a supramolecular interaction of $\beta C D$ and Plasmonic interaction. c) Laser Fluorescence Microscopy Imaging of aggregated nanoparticles. Inset image: Individual nanoparticles detection. Reprinted with permission from Ref 89 (Guillermo Bracamonte et al.). Copyright 2018 J. of Nanophotonics

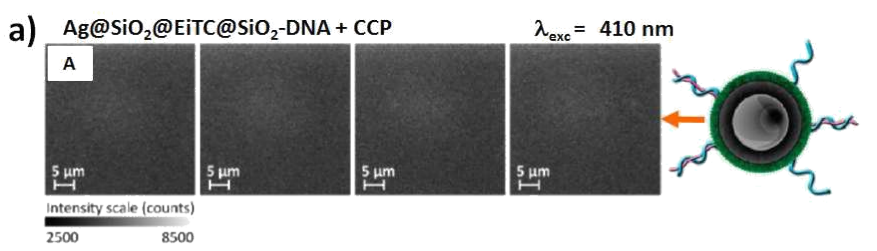

b) $\mathrm{Ag} @ \mathrm{SiO}_{2} @ E \mathrm{EiTC} @ \mathrm{SiO}_{2}-\mathrm{DNA}+\mathrm{CCP}+\mathrm{DNA}$ target $\lambda_{\mathrm{exc}}=410 \mathrm{~nm}$

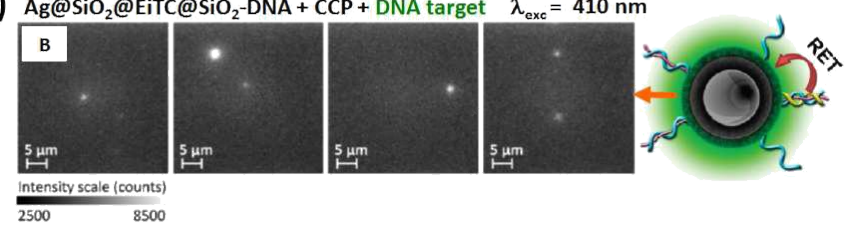

Figure 5. Genotyping based on Imaging Flow Cytometry (IFC): Fluorescence NanoImaging recorded at the nominal emission wavelength of the eosin emitter as energy acceptor of (A) target ready Nanoparticles without complementary targets; and of (B) target Nanoparticles with the addition of complementary targeted DNA strands. Reprinted with permission from Ref 90 (Denis Boudreau et al.). Copyright 2011 ACS Nano

\section{Detection Diagnostics Nanomedicine

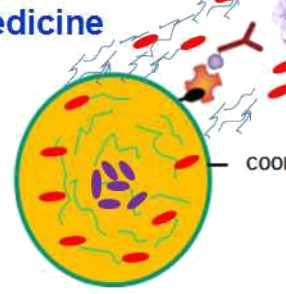 \\ Nanoparticles (NPs)
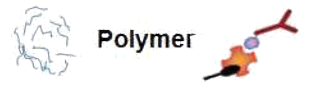 \\ Recognition sites Antibodies

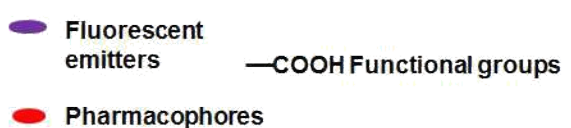 \\ Biological structure}

Figure 6. Schematic representation of Cargo loaded nanoparticles for tracking and drug delivery applications

\section{Future perspectives}

Many challenges should be taken into account within this field from different points of view in order to develop new Nanomaterials joining multidisciplinary knowledge. By this manner it should be highlighted the control of the size at the Nanoscale accompanied with higher intensities from reduced surfaces for Nanoparticle tracking and improved Biostructures resolution. Developments of Nanoplatforms for Single Molecule Detection (SMD); coupled to In Flow methodologies and Optical detection based on Imaging.

For Advanced Nanomedicine, to prevent health problems as well as to improve the healthy population, by Precise and personalized treatments it is essential to develop Nano-platforms for Genomics and Epigenetics diagnosis based on Biophotonics and Bioimaging. And at this point it should be highlighted the early detection of gene expression based non-canonical 3D forms and Epigenetics.

Moreover, for in-vivo applications Biocompatible Hybrids Nanomaterials with tunable properties for drug loading and smart responsive controlled delivery are vital for Cargo Nanoarchitectures for enzyme, and Biomolecule targeted administration applied for specific treatments. In addition, Multifunctional nanoparticles and Multilayered nanoparticles in order to afford diverse targeted functions 
accompanied with smart responsive actions at the different steps of the treatments are highly desired. And, it should be highlighted as well the application of Nanoarchitectures for improve health and well-being as prevention for the healthy population as well accompanied with physical activities that it could be even being applied within Biopharma and Nutraceutics products.

\section{Acknowledgments}

We would like to gratefully acknowledge the different Grants received in order to accomplish the Research work in progress of the authors in this field, CONICET, Consejo Nacional de Investigaciones Científicas y Técnicas (National Research Council of Argentine); ANPCyT, Agencia Nacional de Promoción Científica y Tecnológica (National Agency of Scientific and Technology Promotion of Argentine); and especially to SECyT (Secretary of Science and Technology from the National University of Cordoba (UNC), Argentina, for awarding us the extension of the Grant for Young Researchers at INFIQC.

Moreover, especially thanks to Professor Denis Boudreau from Département de chimie and Centre d'optique, photonique et laser (COPL), Québec, Canada, for the Research Collaboration in progress; as well as specially thanks to all the Canadian Grants that permit it.

Finally specially thanks to Lucia V. Bracamonte and Rosalia F. Bracamonte for the English revision.

\section{References}

1. Nguyen HL, Nguyen HN, Nguyen HH, Luu MQ, Nguyen MH (2015) Nanoparticles: synthesis and applications in life science and environmental technology, Advances in Natural Sciences: Nanoscience and Nanotechnology. Adv Nat Sci: Nanosci. Nanotechnol 6: 1-9.

2. Gontero D, Lessard-Viger M, Brouard D, Bracamonte AG, Boudreau D, et al. (2017) Smart Multifunctional Nanoparticles design as Sensors and Drug delivery systems based on Supramolecular chemistry. Microchemical Journal 130: 316-328.

3. Lackowicz JR (2005) Radiative decay engineering: Metal enhanced fluorescence and plasmon emission, Analytical Biochemistry 337: 171-194. [Crossref]

4. Viger ML, Live LS, O.D. Therrien, D. Boudreau, Reduction of Self-Quenching in Fluorescent Silica-Coated Silver Nanoparticles. Plasmonics 3: 33-40.

5. Geddes CD1 (2013) Metal-enhanced fluorescence. Phys Chem Chem Phys 15: 19537. [Crossref]

6. Brent M. DeVetter Bruce E. Bernacki Wendy D. Bennett Alan Schemer-Kohrn Kyle J. Alvine, Multiresonant layered plasmonic films, Journal of Nanophotonics 11:1-8.

7. Luchowski R, Calander N, Shtoyko T, Apicella E, Borejdo J, et al. (2010) Plasmonic platforms of self-assembled silver nanostructures in application to fluorescence. $J$ Nanophotonics 22: 4 [Crossref]

8. Li JF, Li CY, Aroca RF (2017) Plasmon-enhanced fluorescence spectroscopy. Chem Soc Rev 46: 3962-3979. [Crossref]

9. Bracamonte AG, Brouard D, Lessard-Viger M, Boudreau D, Veglia AV (2016) Nanosupramolecular complex synthesis: switch on/off enhanced fluorescence control an molecular release using a simple chemistry reaction, Microchemical Journal 128: 297304.

10. Puchkova A, Vietz C, Pibiri E, Wünsch B, Sanz Paz M, et al. (2015) Tinnefeld, DNA origami nanoantennas with over 5000-fold fluorescence enhancement and singlemolecule detection at $25 \mu \mathrm{M}$, nanoletters Nano Lett 15: 8354-8359. [Crossref]

11. Aslan K, Gryczynski I, Malicka J, Matveeva E, Lakowicz JR, et al. (2005) Metalenhanced fluorescence: an emerging tool in biotechnology. Curr Opin Biotechnol 16: 55-62. [Crossref]

12. Stoddart JF (2009) The master of chemical topology. Chem Soc Rev 38: 1521-1529. [Crossref]

13. Ambrogio MW, Pecorelli TA, Patel K, Khashab NM, Trabolsi A, et al. (2010) Snap-top nanocarriers. Org Lett 12: 3304-3307. [Crossref]
14. Hell SW (2015) Nanoscopy with focused light (Nobel Lecture). Angew Chem Int Ed Engl 54: 8054-8066. [Crossref]

15. Jeet J, Kobo A, Su T, Grunwald A, Ori Green, et al. (2016) Super-resolution genome mapping in silicon nanochannels. ACS Nano 10: 9823-9830.

16. Dore K, Leclerc M, Boudreau D (2006) Investigation of a fluorescence signal amplification mechanism used for the direct molecular detection of nucleic acids. $J$ Fluoresc 16: 259-265.

17. Armitage BA (2005) Cyanine Dye-DNA interactions: intercalation, groove binding, and aggregation. Top Curr Chem 253: 55-76.

18. Vijay P, McIntyre AB, Mason CE, Greenfield JP, Li S (2016) Clinical genomics challenges and opportunities. Crit Rev Eukaryot Gene Expr 26: 97-113. [Crossref]

19. Hodson R (2016) Precision medicine. Nature 537: S49. [Crossref]

20. Vijay P, McIntyre ABR, Mason CE, Greenfield JP, Li S, (2016) Clinical Genomics: Challenges and Opportunities, Crit Rev Eukaryot Gene Expr 26: 97-113.

21. Carell T, Kurz MQ, Müller M, Rossa M, Spada F (2018) Non-canonical Bases in the Genome: The Regulatory Information Layer in DNA. Angew Chem Int Ed Engl 57: 4296-4312. [Crossref]

22. N.Talebi (2017), Electrons interacting with metamaterials: from few-photon sources to electron optics, SPIE Newsroom. Nanotechnology 1-3.

23. Suh WH, Suslick KS, Stucky GD, Suh YH (2009) Nanotechnology, nanotoxicology, and neuroscience. Prog Neurobiol 87: 133-170. [Crossref]

24. Meziani MJ, Bunker CE, Lu F, Li H, Wang W, et al. (2009) Formation and properties of stabilized aluminum nanoparticles. ACS Appl Mater Interfaces 1: 703-709. [Crossref]

25. Meshgi MA, Kriechbaum M, Biswas S, Holmes D, Marschner C (2016) Synthesis of indium nanoparticles at ambient temperature; simultaneous phase transfer and ripening, J Nanopart Res 18: 363-367.

26. Khan A, Rashid A, Younas R, Chong R (2016) A chemical reduction approach to the synthesis of copper nanoparticles, Int Nano Lett 6: 2126.

27. Enustun BV, Turkevich J (1963) Coagulation of Colloidal Gold, Journal of American Chem. Society 85, 21: 3317-3328.

28. Kimling J, Maier M, Okenve B, Kotaidis V, Ballot H, et al. (2006) Turkevich method for gold nanoparticle synthesis revisited. J Phys Chem B 110: 15700-15707. [Crossref]

29. Murcia MJ (2007), Naumann CA, Chapter 1: Biofunctionalization of fluorescent nanoparticles, nanotechnologies for the life.

30. Kainthan RK, Janzen J, Levin E, Devine DV, Brooks DE, Biocompatibility testing of branched and linear polyglycidol. Biomacromolecules 7: 703-709.

31. Lehn JM, Angew. Chem. Int. Ed. Engl 2,71 (1988) 89-112.

32. Anslyn EV (2007) Supramolecular analytical chemistry. J Org Chem 72: 687-699. [Crossref]

33. Serpell CJ, Cookson J, Ozkaya D, Beer PD (2011) Core@shell bimetallic nanoparticle synthesis via anion coordination, Nature Chemistry 3: 478-483.

34. Viger ML, Live LS, Therrien OD, Boudreau D (2008) Reduction of Self-Quenching in Fluorescent Silica-Coated Silver Nanoparticles, Plasmonics, 3: 33-40.

35. Vowinkel S, Scha fer CG, Cherkashinin G, Fasel C, Roth, et al. (2016) 3D-ordered carbon materials by melt-shear organization for tailor-made hybrid core-shell polymer particle architectures, J. Mater. Chem. C, 4: 3976-3986.

36. Lakowicz JR, Ray K, Chowdhury M, Szmacinski H, Fu Y, et al. (2008) Plasmoncontrolled fluorescence: a new paradigm in fluorescence spectroscopy. Analyst 133: 1308-1346. [Crossref]

37. Asselin J, Lambert MP, Fontaine N, Boudreau D (2017) A ratiometric nanoarchitecture for the simultaneous detection of $\mathrm{pH}$ and halide ions using UV plasmon-enhanced fluorescence. Chem Commun (Camb) 53: 755-758. [Crossref]

38. Rioux M, Gontero D, Veglia AV, Bracamonte AG, Boudreau D (2017) Synthesis of Ultraluminiscent gold core-shell Nanoparticles as NanoImaging Platforms for Biosensing applications based on Metal enhanced fluorescence. RSC Adv 7: 1025210258

39. Fahmy TM, Fong PM, Park J, Constable T, Saltzman WM (2007) Nanosystems for simultaneous imaging and drug delivery to T cells. AAPS J 9: E171-E180. [Crossref]

40. Björnmalm M, Thurecht KJ, Michael M, Scott AM, et al. (2017) Bridging Bio-Nano Science and Cancer Nanomedicine. ACS Nano 11: 9594-9613. [Crossref] 
41. Xu J, Yu M, Peng C, Carter P, Tian J, et al. (2018) Dose Dependencies and Biocompatibility of Renal Clearable Gold Nanoparticles:From Mice to Non-human Primates, Angew Chem Int Ed 57: 266-27.

42. Chen PC, Mwakwari SC, Oyelere AK (2008) Gold nanoparticles: From nanomedicine to nanosensing. Nanotechnol Sci Appl 1: 45-65. [Crossref]

43. Losasso C, Belluco S, Cibin V, Zavagnin P, Micetic I, et al. (2014) Antibacterial activity of silver nanoparticles: sensitivity of different Salmonella serovars. Front Microbiol 5: 227. [Crossref]

44. Gaumet M, Vargas A, Gurny R, Delie F (2008) Nanoparticles for drug delivery: the need for precision in reporting particle size parameters. Eur J Pharm Biopharm 69: 1-9. [Crossref]

45. Yi Y, Kim JH, Kang HW, Oh HS, Kim SW, et al. (2005) A polymeric nanoparticle consisting of mPEG-PLA-Toco and PLMA-COONa as a drug carrier: improvements in cellular uptake and biodistribution, Pharm Res 22: 200-8. [Crossref]

46. Li B, Xie J, Yuan Z, Jain P, Lin Xet al. (2018) Mitigation of Inflammatory Immune Responses with Hydrophilic Nanoparticles Angewandte. Chem. Int. Ed 57: 4527-4531.

47. Davis ME, Zuckerman JE (2010) Evidence of RNAi in humans from systemically administered siRNA via targeted nanoparticles . Choi Nature 464: 1067.

48. International Human Genome Sequencing Consortium, Initial sequencing and analysis of the human genome. Nature 409: 861-921.

49. Vijay P, McIntyre AB, Mason CE, Greenfield JP, Li S (2016) Clinical Genomics: Challenges and Opportunities. Crit Rev Eukaryot Gene Expr 26: 97-113. [Crossref]

50. Venter JC, Adams MD, Myers EW, Li PW, Mural RJ, et al. (2001) The sequence of the human genome. Science 291: 1304-1351. [Crossref]

51. Klosin A, Reis K, Hidalgo-Carcedo C, Casas E, Vavouri T, et al. (2017) Imapred DNA replication derepreses chromatin and genertaes a transgenerationally inherited epigenetic memory. Sciences Advances 3: 1-10.

52. Liang P, Xu Y, Zhang X, Ding C, Huang R, et al. (2015) CRISPR/Cas9-mediated gene editing in human tripronuclear zygotes. Protein Cell 6: 363-372.

53. Liang P, Ding C, Sun H, Xie X, Xu Y, et al. (2017) Correction of ß-thalassemia mutant by base editor in human embryos Protein Cell 8: 811-822.

54. Wang P, Zhang L, Zheng W, Cong L, Guo Z, et al. (2018) Thermo-triggered Release of CRISPR-Cas9 System by Lipid-Encapsulated Gold Nanop articles for Tumor Therapy, Angew. Chem. Int.Ed 57: 1491-1496.

55. Mattar R, de Campos DF, Carrilho MFJ (2012) Lactose intolerance: diagnosis, genetic, and clinical factors Clinical and Experimental Gastroenterology 5: 113-121.

56. Thompson PM, Martin NG, Wright MJ (2010) Imaging genomics. Curr Opin Neurol 23: 368-373. [Crossref]

57. Lakowicz JR (2006) Principles of Fluorescence Spectroscopy, Book Chapter 15: Energy Transfer to Multiple Acceptors in One,Two,or Three Dimensions. Springer Boston MA, 507-528.

58. I. Meglinski (2015) Biophotonics for Medical Applications, Woodhead Publishing Series in Biomaterials, Book 1st Edition.

59. Viger ML, Brouard D, Boudreau D (2011) Plasmon-enhanced resonance energy transfer from a conjugated polymer to fluorescent multilayer core-shell nanoparticles: a photophysical study. J Phys Chem C 115: 2974-2981.

60. Zhang J, Fu Y, Chowdhury MH, Lakowicz JR (2007) Metal-enhanced single-molecule fluorescence on silver particle monomer and dimer: coupling effect between metal particles. Nano Lett 7: 2101-2107.

61. Kodaimati MS, Wang C, Chapman C, Schatz GC (2017) Distance-dependence of interparticle energy transfer in the near-infrared within electrostatic assemblies of $\mathrm{PbS}$ quantum dots ACS Nano 11: 5041-5050.

62. Garcia-Vidal FJ, Feis J (2017) Long-distance operator for energy transfer Nanophotonics can be used to enhance the electromagnetic coupling between molecules. Science 357: 1357-1358.

63. Sekar RB, Periasamy A (2003) Fluorescence resonance energy transfer (FRET) microscopy imaging of live cell protein localizations, The Journal of Cell Biology 160: 629-633.

64. Chan FKM (2004) Monitoring Molecular Interactions inLiving Cells Using Flow Cytometr ic Analysis of Fluorescence Resonance Energy Transfer. Methods Mol Biol 261: 371-381
65. Margineanu A, Chan JJ, Kelly DJ, Warren SC, Flatters D, et al. (2016) Screening for protein-protein interactions using Förster resonance energy transfer (FRET) and fluorescence lifetime imaging microscopy (FLIM). Nature Scientific Reports 6: 1-15.

66. Shchukina AL, Osadko IS, Eremchev IY (2013) Statistics for fluorescence photons of donor and acceptor molecules involved in non-radiative energy transfer (FRET). Journal of Physics 478: 1-10.

67. Puchkova A, Vietz C, Pibiri E, Wunsch B, Sanz Paz M, et al. (2015) DNA Origami Nanoantennas with over 5000-fold Fluorescence Enhancement and Single-Molecule Detection at $25 \mu \mathrm{M}$. Nano Lett 15: 8354-8359. [Crossref]

68. Palecek D, Edlund P, Westenhoff S, Zigmantas D (2017) Quantum coherence as a witness of vibronically hot energy transfer in bacterial reaction center. Science Advances 3: 1-6.

69. Tang B, Cao L, Xu K, Zhuo L, Ge J, A New Nanobiosensor for Glucose with High Sensitivity and Selectivity in Serum Based on Fluorescence Resonance Energy Transfer (FRET) between CdTe Quantum Dots and Au Nanoparticles, Chem. Eur 14: $3637-3644$

70. Shu D, Zhang H, Petrenko R, Meller J, Guo P (2010) Dual-Channel Single-Molecule fluorescence Resonance Energy Transfer to Establish Distance Parameters for RNANano particles, ACS NANO, 4: 6843-6853.

71. Jepsen MDE, Sparvath SM, Nielsen TB, Langvad AH, Grossi G, et al. (2018) Development of a genetically encodable FRET system using fluorescent RNA aptamers. Nature Communications 9: 1-4

72. Zhao W, Li M, Chang CZ, Jiang J, Wu L, et al. (2018) Direct imaging of electron transfer and its influence on superconducting pairing at $\mathrm{FeSe} / \mathrm{SrTiO} 3$ interface. Science Advances 16 1-7. [Crossref]

73. Asselin J, Legros P, Grégoire A, Boudreau D (2016) Correlating Metal-Enhanced Fluorescence and Structural Properties in $\mathrm{Ag} @ \mathrm{SiO} 2$ Core-Shell Nanoparticles, Plasmonics 1-8.

74. Lackowicz JR (2005), Radiative decay engineering: Metal enhanced fluorescence and plasmon emission. Analytical Biochemistry 337: 171-194.

75. Ray K, Chowdhury MH, Szmacinski H, Lakowicz JR (2008) Metal-Enhanced Intrinsic Fluorescence of Proteins on Silver Nanostructured Surfaces toward Label-Free Detection. J. Phys. Chem 46: 17957-17963.

76. Lessard-Viger M, Brouard D, Boudreau D (2011) Plasmon Enhanced energy transfer from a conjugated polymer to Fluorescent core shell Nanoparticles: A photophysical study. J. Phys. Chem 115: 2974-2981.

77. Graves EE, Yessayan D, Turner G, Weissleder R, Ntziachristos V (2005) Validation of in vivo fluorochrome concentrations measured using fluorescence molecular tomography. J Biomed Opt 10: 44019. [Crossref]

78. Haam (2007) Antibody conjugated magnetic PLGC nanoparticles for diagnosis and treatment of breast cancer. J Mater Chem 17: 2695-2699.

79. Wagner M, Horn M, Daims H (2003) Fluorescence in situ hybridisation for the identification and characterisation of prokaryotes. Curr Opin Microbiol 6: 302-309. [Crossref]

80. Dubertret B, Skourides P, Norris DJ, Noireaux V, Brivanlou AH, et al. (2002) In vivo imaging of quantum dots encapsulated in phospholipid micelles. Science 298: 17591762. [Crossref]

81. Jeffet J, Kobo A, Su T, Grunwald A, Green O, et al. (2016) Super-Resolution Genome Mapping in Silicon Nanochannels. ACS Nano 10: 9823-9830. [Crossref]

82. Hikage M, Gonda K, Takeda M, Kamei T, Kobayashi M, et al. (2010) Nano-imaging of the lymph network structure with quantum dots. Nanotechnology 21: 185103. [Crossref]

83. Pompa P, Martiradonna L, Torre AD, Sala FD, Manna L, et al. (2006) MEFof colloidal nanocrystals with nanoscale control. Nature Nanotechnology 1: 126-130.

84. Magnan F, Gagnon J, Fontaine FG, Boudreau D (2013) Indium@silica coreshell nanoparticles as plasmonic enhancers of molecular luminescence in the UV region. Chem Commun (Camb) 49: 9299-9301. [Crossref]

85. Asselin J, Viger ML, Boudreau D, Review Article, Metal-Enhanced Fluorescence and FRET in Multilayer Core-Shell Nanoparticles, Advances in Chemistry. Hindawi Publishing Corporation 812313: 1-16.

86. Gregoire A, Boudreau D, Chapter 28: Metal-Enhanced Fluorescence in Plasmonic Waveguides, Springer Science+Business Media Dordrecht 2017 B. Di Bartolo et al. (eds.), Nano-Optics: Principles Enabling Basic Research and Applications, NATO Science for Peace and Security Series B: Physics and Biophysics. 
87. Golberg K, Elbaz A, McNeil R, Kushmaro A, Geddes CD, et al. (2014) Increased Bioassay Sensitivity of Bioactive Molecule Discovery using Metal-Enhanced Bioluminescence. Journal of Nanoparticle Research 16: 2770-2775.

88. Gontero D, Rioux M, Veglia AV, Boudreau D, Bracamonte AG, "Ultraluminescent gold Core@shell nanoparticles applied to individual bacterial detection based on Metal-Enhanced Fluorescence Nanoimaging", J. of Nanophotonics. Special issue Nanoplasmonics for Biosensing, Enhanced Light-Matter Interaction, and Spectral Engineering 12: 1-12.

89. Veglia AV, Bracamonte AG (2018) Metal Enhanced fluorescence emission and Quenching protection effect with a host-guest Nanophotonic-supramolecular structure. J. Nanophoton 12: 1-12.

90. Brouard D, Viger ML, Bracamonte AG, Boudreau D (2011) Label-free biosensing based on multilayer fluorescent nanocomposites and a cationic polymeric transducer. ACS Nano 5: 1888-1896.

91. Brouard D, Ratelle O, Bracamonte AG, St-Louis M, Boudreau D (2013) Direct molecular detection of SRY gene from unamplified genomic DNA by metal-enhanced fluorescence and FRET. Analytical Methods 5: 6896-6899.

92. Brouard D, Ratelle O, Perreault J, Boudreau D, St-Louis M (2014) PCR-free blood group genotyping using a nanobiosensor, Vox Sanguinis, International Society of Blood Transfusion, 1-8. DOI: $10.1111 /$ vox.12207.

93. Gruber B, Stadlbauer S, Spath A, Weiss S, Kalinina M, et al. (2010) Modular Chemosensors from Self-Assembled Vesicle Membranes with Amphiphilic Binding Sites and Reporter Dyes. Angew. Chem. Int. Ed 49: 7125-7128.
94. Muller A, Konig B (2014) Vesicular aptasensor for the detection of thrombin. Chem Commun (Camb) 50: 12665-12668. [Crossref]

95. Guo J, Hendrickson JR, Luk TS (2018) Special Section Guest Editorial: Nanoplasmonics for Biosensing and Enhanced Light-Matter Interaction. J. Nanophoton 12

96. Duan Y, Ye F, Huang Y, Qin Y, He, et al (2018) One-pot synthesis of a metal-organic framework-based drug carrier for intelligent glucose-responsive insulin delivery. Chem. Commun 54: 377-5380.

97. Yahia-Ammar A, Sierra D, Merola F, Hildebrandt N, Le Guevel X (2016) SelfAssembled Gold Nanoclusters for Bright Fluorescence Imaging and Enhanced Drug Delivery. ACS Nano 10: 2591-2599. [Crossref]

98. Yang J, Lee CH, Park J, Seo S, Lim EK, et al. (2007) Haam; Antibody conjugated magnetic PLGA nanoparticles for diagnosis and treatment of breast cancer. J. Mater Chem 17: 2695-2699.

99. Evans CL, Chan KF, Prow T, Osseiran S (2018) Visualizing and quantifying drug uptake in skin, Nwesroom SPIE, 10.1117/2.1201705.006809 1-3.

100. Genevois C, Loiseau H, Couillaud F (2016) In Vivo Follow-up of Brain Tumor Growth via Bioluminescence Imaging and Fluorescence Tomography. Int $\mathrm{J} \mathrm{Mol}$ Sci 17. [Crossref]

101. Dufour S, De Koninck Y (2015) Optrodes for combined optogenetics and electrophysiology in live animals. Neurophotonics 2: 031205. [Crossref]

Copyright: ( 02018 Salinas CI. This is an open-access article distributed under the terms of the Creative Commons Attribution License, which permits unrestricted use, distribution, and reproduction in any medium, provided the original author and source are credited. 